IOS Press

\title{
Neutrophil/lymphocyte ratio helps select metastatic pancreatic cancer patients benefitting from oxaliplatin
}

\author{
Vincenzo Formica ${ }^{\mathrm{a}, *}$, Cristina Morelli $^{\mathrm{a}}$, Patrizia Ferroni ${ }^{\mathrm{b}, \mathrm{c}}$, Antonella Nardecchia $^{\mathrm{a}}$, Manfredi Tesauro ${ }^{\mathrm{d}}$, \\ Stefania Pellicori ${ }^{\mathrm{a}}$, Vittore Cereda ${ }^{\mathrm{a}}$, Antonio Russo ${ }^{\mathrm{e}}$, Silvia Riondino $^{\mathrm{a}, \mathrm{c}}$, Fiorella Guadagni ${ }^{\mathrm{b}, \mathrm{c}}$ and \\ Mario Roselli ${ }^{\mathrm{a}}$ \\ ${ }^{a}$ Department of Systems Medicine, Medical Oncology, Tor Vergata Clinical Center, Tor Vergata University of \\ Rome, Rome, Italy \\ ${ }^{\mathrm{b}}$ San Raffaele Roma Open University, Rome, Italy \\ ${ }^{\mathrm{C}}$ Interinstitutional Multidisciplinary Biobank (BioBIM), IRCCS San Raffaele Pisana, Rome, Italy \\ ${ }^{\mathrm{d}}$ Department of Systems Medicine, Internal Medicine, Tor Vergata Clinical Center, Tor Vergata University of \\ Rome, Rome, Italy \\ e Section of Medical Oncology, Department of Surgical and Oncology Sciences, University of Palermo, Italy
}

\begin{abstract}
.
BACKGROUND: High Neutrophil/Lymphocyte ratio (NLR), as a measure of enhanced inflammatory response, has been negatively associated with prognosis in patients with localized pancreatic ductal adenocarcinoma (PDA).

OBJECTIVE: In the present study, we aimed at investigating the prognostic value of NLR in two homogeneous groups of chemotherapy-naïve metastatic PDA patients. Patients were treated with either gemcitabine (GEM) or gemcitabine/ oxaliplatin (GEMOXA). We also assessed whether NLR could identify patients benefiting from the use of oxaliplatin.

METHODS: Consecutive PDA patients treated at the Medical Oncology Unit of Tor Vergata University Hospital of Rome with either GEM or GEMOXA were included ( $n=103$ ). NLR was assessed before and during chemotherapy and correlated with outcome together with common clinical and biochemical variables.

RESULTS: Among 17 analyzed variables NLR, Karhofsky Perfomance Status (KPS), d-dimer and erythrocyte sedimentation rate were found to be significantly associated with median Overall Survival (mOS) at the univariate analysis. Only NLR and KPS were independent prognosticator at multivariate analysis, with NLR displaying the highest statistical significance. NLR was also predictive of oxaliplatin activity, as only patients with NLR $>2.5$ (cutoff determined upon ROC analysis) derived benefit from GEMOXA over GEM.

CONCLUSIONS: NLR is both an independent prognostic and predictive factor in metastatic PDA, since only patients with high NLR seem to benefit from the addition of oxaliplatin. NLR may help select patients for whom a particularly poor prognosis might justify more intensive, yet less tolerable, combination regimens.
\end{abstract}

Keywords: Neutrophil/lymphocyte ratio, pancreatic ductal adenocarcinoma, gemcitabine, oxaliplatin

\section{Introduction}

${ }^{*}$ Corresponding author: Vincenzo Formica, Department of Systems Medicine, Medical Oncology, Tor Vergata Clinical Center, Tor Vergata University of Rome, Viale Oxford, 81-00133 Rome, Italy. Tel.: +39 0620908190; Fax: +39 0620903806; E-mail: vincenzo.formica@uniroma2.it.

Pancreatic ductal adenocarcinoma (PDA) is one of the most aggressive tumors, with dismal prognosis.

At the time of diagnosis, most patients present with inoperable disease and have a median survival of 6 to 8 months [1]. 
For unresectable patients, systemic chemotherapy is the standard. Until recently, guidelines have recommended gemcitabine (GEM) as the standard first-line treatment, because of its efficacy and its manageable toxicity also in frail subjects [2]. Up to 2011, polychemotherapy regimens could not demonstrate a major advantage in survival over gemcitabine monotherapy, even in large randomized phase III trials and, therefore, were not used as a standard [3-12].

However, a meta-analysis published in 2007 found a small but significant benefit from the addition of platinum compounds or fluorouracil to gemcitabine [13], and in some centers GEM-based combinations (e.g. gemcitabine + oxaliplatin (GEMOXA), gemcitabine + cisplatin, gemcitabine + fluoropyrimidines, gemcitabine+erlotinib) have been considered as a possible choice for patients with good performance status (PS) and the need for a rapid radiological response $[2,13]$.

In 2011, results from the PRODIGE 4/ACCORD 11 trial were published demonstrating for the first time that the three-drug FOLFIRINOX regimen was superior to GEM alone for patients with excellent PS [14].

Lately, the addition of an albumin-bound taxane to gemcitabine was found to produce a $28 \%$ reduction in the risk of death in the MPACT trial [15].

Considering all these findings, GEM alone may be a suboptimal treatment for particularly fit patients. It would be desirable to identify specific pre-treatment prognostic factors able to determine which subgroup of patients would really benefit from more aggressive combination chemotherapy.

Systemic inflammation is a process associated with a number of pathological conditions, such as metabolic disorders [16], atherosclerosis [17], osteoporosis [18] and cancer [19-23]. It might be considered as a doubleedged weapon in that it represents the first line immune defense but also a source of cytokines and growth factors with potentially adverse effects.

Blood neutrophil to lymphocyte ratio (NLR) is a reliable biomarker of systemic inflammatory response. High NLR has been found to have an adverse effect on prognosis for localized pancreatic and periampullary malignancies. A few studies have recently confirmed these results also in the metastatic setting [24].

Owing to the worse reported outcome, PDA patients with high NLR are ideal candidates for more aggressive treatment with gemcitabine-based combination chemotherapy. In an update of the MPACT study, the effect of NLR was investigated, and there appeared to be a higher efficacy of nab-paclitaxel plus gemcitabine regimen in patients with NLR $>5$ [15].
Up to 2011, in our center GEMOXA has been the preferred option for PDA patients with good performance status, because of the convenient schedule (biweekly administration) and the relatively good tolerance

Authors acknowledge that the preference for GEMOXA can be questionable; however results in the present study are hypothesis generating and could be taken into consideration for future studies aiming at identifying predictive markers for other combination regimens.

None of the previous researches have investigated whether NLR is predictive in discriminating patients who would benefit from the GEMOXA doublet over GEM alone.

The aim of the present study was to select two homogenous groups of patients, receiving either GEMOXA or GEM, depending on reasons other than performance status or general health condition. Main reasons of regimen choice were patient preference or pre-existent neuropathy (mainly diabetes-related) indicating oxaliplatin withdrawal. The prognostic and predictive role of NLR was investigated.

\section{Materials and methods}

Among all consecutive patients referred to the Medical Oncology Unit of Tor Vergata University Hospital, patients with histologically confirmed diagnosis of PDA and measurable metastatic disease were eligible for this retrospective observational study. The evaluated referral period was between April 2008 and October 2011. Patients with active infection, autoimmune disease and stable treatment with steroids, were excluded.

Included patients were required to be chemotherapynaïve. Patients had to have Karnofsky PS (KPS) 100 to 80 and adequate hematological $(\mathrm{Hb} \geqslant 9 \mathrm{~g} / \mathrm{dl}$, white blood cell $(\mathrm{WBC}) \geqslant 2000 / \mathrm{mm}^{3}$, platelet count $\geqslant$ $60000 / \mathrm{mm}^{3}$ ), hepatic (bilirubin $\leqslant 1.5 \times$ upper limit of normal (ULN), alanine amino-transferase (ALT) and aspartate aminotransferase $(\mathrm{AST}) \leqslant 1.5 \times \mathrm{ULN})$, and renal (creatinine $\leqslant 1.5 \times \mathrm{ULN}$ ) functions. Patients with poor performance status (KPS $<80$ ) were excluded from the study.

A standard first-line therapy with either GEM (gemcitabine $1000 \mathrm{mg} / \mathrm{m}^{2}$ weekly, 7 weeks on 1 week off for the first cycle, 3 weeks on 1 week off for the following cycles) or GEMOXA (gemcitabine $1000 \mathrm{mg} / \mathrm{m}^{2}$ day 1 , oxaliplatin $100 \mathrm{mg} / \mathrm{m}^{2}$ day 1 , cycles repeated every 2 weeks) was chosen for treatment. 
As per internal guidelines, the preferred regimen for patients with KPS $>80$ was GEMOXA.

However, in some cases the GEM regimen was adopted, with main reasons for withdrawing oxaliplatin being 1) patient's preference (i.e. patients not willing to receive a more toxic regimen in light of a possible minimal improvement in disease outcome) or 2) pre-existent peripheral neuropathy (mainly diabetesrelated).

Treatment was continued until tumor progression, unacceptable toxicity, or request of termination by individual patients.

Assessment of response was performed every 8 weeks of treatment by comparison of a thorax/abdomen/pelvis CT scan, according to RECIST criteria, with the baseline CT scan that had been performed within 45 days prior to chemotherapy initiation.

As per routine practice, the following data were collected in the institutional electronic medical records before treatment commencement: gender, age, body mass index (BMI), KPS, circulating tumor markers (carcinoembryonic antigen (CEA) and carbohydrate antigen 19.9 (CA 19.9)), routine hematology including full blood count (neutrophils, lymphocytes, monocytes, haemoglobin and platelets), biochemical blood tests including Alkaline Phosphatase (ALP), Albuminemia, C-reactive protein (CRP), erythrocyte sedimentation rate (ESR), lactate dehydrogenase (LDH). KPS evaluation, BMI, CEA, CA 19.9, routine hematology and biochemical blood tests were also performed during treatment at each chemotherapy administration (i.e. every week/two weeks). Hematology test allowed NLR calculation. Baseline data were considered evaluable if collected within 3 weeks before the start of the treatment.

All patients signed an informed consent to data analysis for retrospective studies.

Patients out of treatment, in palliative care, but still capable of attending the clinic, were offered to be clinically followed-up every two months at the Medical Oncology Unit Outpatient Service of Tor Vergata University Hospital.

The study was approved by the local ethics committee and Institutional Review Board and therefore carried out in accordance with The Code of Ethics of the World Medical Association (Declaration of Helsinki) for experiments involving humans.

\subsection{Statistical analysis}

The primary endpoint was to evaluate the predictive value of baseline and one-month NLR for Overall
Survival (OS), defined as time from treatment start to death from any cause. If a patient had not died, OS was censored at the time of the last follow-up.

The secondary endpoint was the association between NLR and: (1) progression-free survival (PFS), defined as the period beginning on the date of treatment commencement and ending with the first observation of disease progression or death from any cause, if a patient had not reached the progression endpoint, PFS was censored at the time of the last follow-up; (2) overall RECIST-defined radiological response rate (ORR) divided into two main categories: presence (complete + partial responses) or absence (stable + disease progression) of radiological tumor response.

Another secondary endpoint was the association between known prognostic factors in PDA and OS.

Examples of prognostic factors for PDA are the tumor marker CA19.9, which is proportional to the tumor burden [25], ALP, which is both an inflammatory and cholestasis marker [26], and d-dimer, which is associated with an enhanced coagulative cascade [27].

Associations between prognostic variables and PFS or OS were examined using the Kaplan-Meier method with long rank test and Cox-regression analysis to assess for differences between subgroups and estimation of hazard ratio (HR) and its associated $95 \%$ confidence interval $(95 \% \mathrm{CI})$.

A Cox proportional hazards regression analysis was performed to evaluate the association between selected prognostic factors and the primary endpoint (OS) in multivariable models.

In the multivariate Cox-regression analysis, the enter method was used to enter independent variables into the model (i.e., all variables were entered into the model in one single step without checking for significance). Only variables found significant (i.e. $p$ value $<0.05)$ at the univariate analysis were included in the multivariate model.

Associations between dichotomized prognostic factors and ORR were evaluated by the chi-square test.

A Receiver Operating Curve (ROC) was constructed to set the most discriminatory NLR cut-off, using long vs short-term survival (i.e. $>$ vs $<6$ months) as a classification variable. The choice of using the 6 months value was based on convenience since only three patients among those still alive had a follow-up inferior to 6 months, and a relevant proportion of patients $(38 \%)$ were classified as short-term survivors.

Differences in patient characteristics between NLR subgroups were tested using either chi-square or T test.

All statistical tests were two-sided. Analyses were done using MedCalc for Windows, version 9.5.0.0 (MedCalc Software, Mariakerke, Belgium). 
Table 1

Characteristics of the 103 study patients. Values refer to absolute number (n.) of patients except when otherwise specified. KPS: Karnofsky performance status, GEM: gemcitabine, GEMOXA: gemcitabine+oxaliplatin, WBC: white blood cells, LDH: lactate dehydrogenase, ALP: alkaline phosphatase, C-reactive protein (CRP), erythrocyte sedimentation rate (ESR), body mass index (BMI), $\mathrm{Hb}$ : haemoglobin, NLR: neutrophil-lymphocyte ratio

\begin{tabular}{|c|c|c|}
\hline Characteristic & Category & n. of patients \\
\hline \multicolumn{3}{|l|}{ Clinical } \\
\hline \multirow[t]{2}{*}{ Sex } & Male & 53 \\
\hline & Female & 50 \\
\hline \multirow[t]{2}{*}{ Age } & Median & 67 years \\
\hline & Range & 41-84 years \\
\hline \multirow[t]{3}{*}{ KPS } & 100 & 53 \\
\hline & 90 & 35 \\
\hline & 80 & 15 \\
\hline \multirow[t]{2}{*}{ Type of chemotherapy } & GEM & 46 \\
\hline & GEMOXA & 57 \\
\hline \multirow[t]{2}{*}{ Diabetes at diagnosis } & Yes & 25 \\
\hline & No & 78 \\
\hline \multicolumn{3}{|l|}{ Biochemical } \\
\hline \multirow[t]{2}{*}{$\mathrm{LDH}$} & Median & $305 \mathrm{U} / 1$ \\
\hline & Range & $122-1162 \mathrm{U} / 1$ \\
\hline \multirow[t]{2}{*}{ ALP } & Median & $168 \mathrm{U} / 1$ \\
\hline & Range & $37-2427 \mathrm{U} / 1$ \\
\hline \multirow[t]{2}{*}{ d-dimer } & Median & $340 \mathrm{ng} / \mathrm{mL}$ \\
\hline & Range & $55-13615 \mathrm{ng} / \mathrm{mL}$ \\
\hline \multirow[t]{2}{*}{ CRP } & Median & $19.00 \mathrm{U} / 1$ \\
\hline & Range & $0.01-252.60 \mathrm{U} / 1$ \\
\hline \multirow[t]{2}{*}{ ESR } & Median & $30 \mathrm{~mm} / \mathrm{h}$ \\
\hline & Range & $18-120 \mathrm{~mm} / \mathrm{h}$ \\
\hline \multirow[t]{2}{*}{ Albumin } & Median & $3.5 \mathrm{~g} / \mathrm{dL}$ \\
\hline & Range & $2.0-4.8 \mathrm{~g} / \mathrm{dL}$ \\
\hline \multirow[t]{2}{*}{ CA $19-9$} & Median & $400 \mathrm{U} / \mathrm{ml}$ \\
\hline & Range & $1-100000 \mathrm{U} / \mathrm{ml}$ \\
\hline \multirow[t]{2}{*}{ CEA } & Median & $3 \mathrm{ng} / \mathrm{ml}$ \\
\hline & Range & $0.3-531.7 \mathrm{ng} / \mathrm{ml}$ \\
\hline \multirow[t]{2}{*}{ WBC } & Median & $7.1 * 10^{3} / \mathrm{mm}^{3}$ \\
\hline & Range & $2.1-25.4 * 10^{3} / \mathrm{mm}^{3}$ \\
\hline \multirow[t]{2}{*}{ Neutrophils } & Median & $4.4 * 10^{3} / \mathrm{mm}^{3}$ \\
\hline & Range & $0.5-20.7 * 10^{3} / \mathrm{mm}^{3}$ \\
\hline \multirow[t]{2}{*}{ Lymphocytes } & Median & $1.7 * 10^{3} / \mathrm{mm}^{3}$ \\
\hline & Range & $0.3-5.3 * 10^{3} / \mathrm{mm}^{3}$ \\
\hline \multirow[t]{2}{*}{ NLR } & Median & $2.5 \mathrm{~mm}^{3} / \mathrm{mm}^{3}$ \\
\hline & Range & $0.3-22.8 \mathrm{~mm}^{3} / \mathrm{mm}^{3}$ \\
\hline \multirow[t]{2}{*}{ Monocytes } & Median & $0.5^{*} 10^{3} / \mathrm{mm}^{3}$ \\
\hline & Range & $0.03-2.69 * 10^{3} / \mathrm{mm}^{3}$ \\
\hline \multirow[t]{2}{*}{$\mathrm{Hb}$} & Median & $12.3 \mathrm{~g} / \mathrm{dL}$ \\
\hline & Range & $9.4-16.0 \mathrm{~g} / \mathrm{dL}$ \\
\hline \multirow[t]{2}{*}{ Platlets } & Median & $240 * 10^{3} / \mathrm{mm}^{3}$ \\
\hline & Range & $63-590 * 10^{3} / \mathrm{mm}^{3}$ \\
\hline \multirow[t]{2}{*}{ BMI } & Median & $23 \mathrm{~kg} / \mathrm{m}^{2}$ \\
\hline & Range & $16-38 \mathrm{~kg} / \mathrm{m}^{2}$ \\
\hline
\end{tabular}

\section{Results}

\subsection{Patients' characteristics and treatment delivery}

Among 203 consecutive patients referred to our Unit between April 2008 and October 2011, 103 patients,
50 female and 53 male, were included into the study (Table 1).

All included patients were treated with either GEM $(\mathrm{N}=46)$ or GEMOXA $(\mathrm{N}=57)$ as per protocol for at least two cycles. The median age at baseline was 67 years (range, 41-84). There was no imbalance in KPS between GEM and GEMOXA group (KPS 80 or 90 in $53 \%$ and $42 \%$ of patients, respectively, chi-square $\mathrm{p}$ value $=0.209)$. Median BMI at treatment start was 23 (range 16-34).

Routine biochemical tests, some of which are well recognized prognostic factors, were performed, as per routine practice, at baseline (Table 1) and at each chemotherapy administration. Adverse biochemical features of ALP > ULN (i.e. 129 U/L), CA19.9 > ULN (i.e. $37 \mathrm{U} / \mathrm{mL}$ ), CEA > ULN (i.e. $5 \mathrm{ng} / \mathrm{mL}$ ), were observed in $65 \%, 80 \%$ and $32 \%$ of cases, respectively.

Median NLR was 2.518, with 25 patients having NLR $>5$. The majority of patients $(54 \%)$ had hematology and biochemical tests the day of chemotherapy commencement, just before drug administration, or the day before; only seven patients had blood tests more than ten days prior to the start of treatment.

Detailed demographic and clinical features are summarized in Table 1.

\subsection{GEM vs GEMOXA}

Among the 103 patients selected for the study, median number of treatment cycles administered was 3 (range, 2-12) and 6 (range, 3-13), in the GEM and GEMOXA group, respectively. In the whole population, ORR was $14 \%$, with a disease control rate (DCR, response + stable disease at 3 months) of $38 \%$.

As of May 2013, 93 out of 103 patients had reached the progression endpoint. median PFS was 4 months (95\% CI 3.4-5.5). Eighty-eight patients had died and 15 were alive. Median follow-up of surviving patients was 22 months (range 2-51). Median OS was 7 months (95\% CI 6.1-8.2).

In the whole study cohort, GEMOXA displayed a superior OS as compared to GEM, with an mOS of 8.0 and 5.8 months, respectively, Hazard Ratio, HR 1.46 (95\% CI 0.94-2.25), but this was of borderline statistical significance (p 0.07, Fig. 1).

\subsection{Prognostic value of NLR}

To investigate the prognostic and predictive value of NLR in study patients, a ROC analysis was performed to set the NLR cutoff with the most favourable 


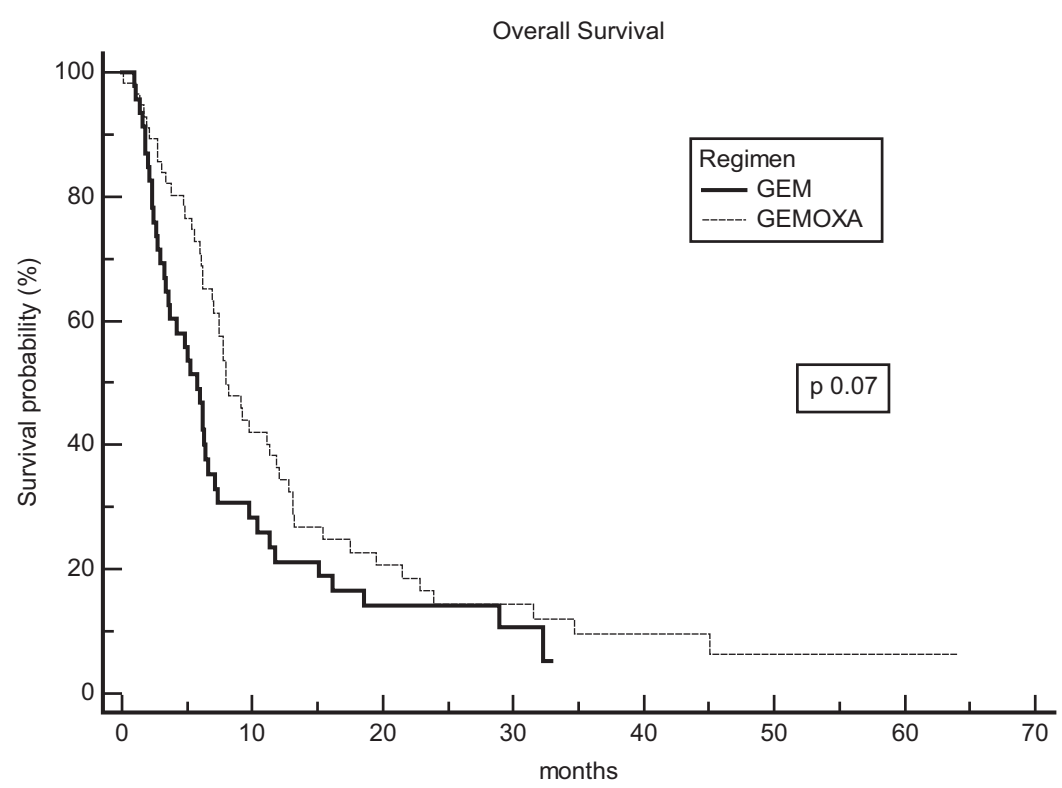

Fig. 1. Overall Survival and first-line chemotherapy regimen in 103 analysed patients. GEM: gemcitabine monotherapy. GEMOXA; gemcitabine plus oxaliplatin.

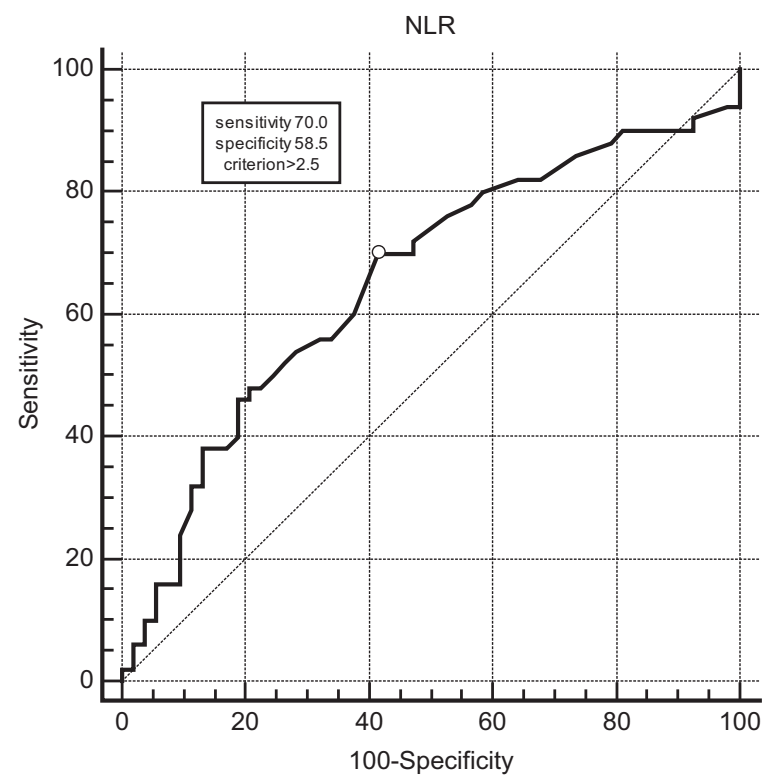

Fig. 2. Receiver Operating Curve (ROC) analysis to set best neutrophil-lymphocyte ratio (NLR) cutoff.

specificity and sensitivity (Fig. 2). Overall survival was used as the classification variable by categorizing patients as long (more than 6 months) and short-term $(<$ 6 months) survivors. The value of $2.5(<2.5 \mathrm{vs}>2.5)$ was found to be the ideal cutoff to distinguish short vs long survivors with $70 \%$ sensitivity and $58.5 \%$ specificity.
Using the cutoff value derived from ROC analysis, NLR was confirmed to be a biomarker significantly associated with overall survival in the whole study cohort, with mOS of 9.2 and 6.0 months for patients with NLR $<$ vs $>2.5$, respectively, HR 1.95 (95\%CI 1.28-2.97), p 0.001. The prognostic power of NLR was also demonstrated with regard to PFS: mPFS 6.4 and 3.4 months, respectively, HR 2.39 (95\%CI 1.57-3.62), $p<0.0001$.

As compared to low NLR patients, high NLR patients had higher baseline d-dimer (mean 1228 vs $383 \mathrm{ng} / \mathrm{mL}$, p 0.01), monocytes (mean 0.75 vs $0.53 * 103 / \mathrm{mm}^{3}, \mathrm{p} 0.02$ ) and ESR (mean $38 \mathrm{vs} 30 \mathrm{~mm} /$ $\mathrm{h}, \mathrm{p}$ 0.03). No other significant differences were detected (in particular no difference in diabetes incidence between high vs low NLR) [28].

\subsection{NLR and other prognostic factors}

To evaluate NLR in conjunction with other clinical and biochemical variables potentially influencing prognosis, univariate and multivariate Cox-regression analyses for the primary endpoint OS were performed (Table 2). At univariate analysis, NLR taken as a continuous variable was confirmed to be significantly associated with survival, with a $13 \%$ increased risk of death per 1-unit increase in NLR $=\exp (\mathrm{b}) 1.13,95 \%$ CI 1.06-1.20, p 0.0001. D-dimer (continuous variable), ESR (continuous variable) and KPS (80 or 90 vs 
Table 2

Univariate and Multivariate Cox-regression analysis for Overall Survival. Only variables with $p<0.05$ at univariate analysis were run in the multivariate model. KPS: Karnofsky performance status, GEM: gemcitabine, GEMOXA: gemcitabine + oxaliplatin, WBC: white blood cells, LDH: lactate dehydrogenase, ALP: alkaline phosphatase, C-reactive protein (CRP), erythrocyte sedimentation rate (ESR), body mass index (BMI), Hb: haemoglobin, NLR: neutrophil/lymphocyte ratio, b: cox-regression coefficient of the relevant variable, SE: standard error for b, Wald: Wald statistic result to derive p-value, Exp: exponential, CI: Confidence Interval. Variables are taken as continuous unless otherwise specified (i.e. x-value vs y-value shape). $\operatorname{Exp}(\mathrm{b})$ represents the Hazard Ratio (HR). For continuous variables, HR refers to the hazard of death for each 1-unit increase of the variable itself

\begin{tabular}{|c|c|c|c|c|c|c|}
\hline Covariate & $\mathrm{B}$ & SE & Wald & $\operatorname{Exp}(b)$ & $95 \%$ CI of $\operatorname{Exp}(b)$ & $P$-value \\
\hline \multicolumn{7}{|l|}{ Univariate } \\
\hline NLR & 0.1198 & 0.0303 & 15.6696 & 1.1272 & 1.0627 to 1.1958 & 0.0001 \\
\hline d-dimer & 0.0002 & 0.0001 & 9.8298 & 1.0002 & 1.0001 to 1.0003 & 0.0017 \\
\hline KPS (80-90 vs 100) & 0.6143 & 0.2243 & 7.5043 & 1.8484 & 1.1937 to 2.8623 & 0.0062 \\
\hline ESR & 0.0160 & 0.0068 & 5.5764 & 1.0161 & 1.0028 to 1.0296 & 0.0182 \\
\hline GEM vs GEMOXA & 0.3857 & 0.2173 & 3.1510 & 1.4706 & 0.9627 to 2.2463 & 0.0759 \\
\hline Sex (female $v s$ male) & 0.3845 & 0.2167 & 3.1491 & 1.4689 & 0.9627 to 2.2413 & 0.0761 \\
\hline ALP & 0.0005 & 0.0004 & 1.4542 & 1.0005 & 0.9997 to 1.0014 & 0.2279 \\
\hline BMI & -0.0332 & 0.0295 & 1.2697 & 0.9673 & 0.9133 to 1.0246 & 0.2598 \\
\hline Albumin & -0.2108 & 0.1894 & 1.2394 & 0.8099 & 0.5599 to 1.1717 & 0.2656 \\
\hline Monocytes & -0.2089 & 0.2429 & 0.7399 & 0.8114 & 0.5053 to 1.3031 & 0.3897 \\
\hline Platelets & -0.0009 & 0.0010 & 0.7313 & 0.9991 & 0.9971 to 1.0011 & 0.3925 \\
\hline CA $19-9$ & 0.0000 & 0.0000 & 0.6821 & 1.0000 & 1.0000 to 1.0000 & 0.4089 \\
\hline $\mathrm{Hb}$ & -0.0635 & 0.0877 & 0.5240 & 0.9385 & 0.7910 to 1.1135 & 0.4692 \\
\hline Diabetes (yes vs no) & 0.1047 & 0.2473 & 0.1794 & 1.1104 & 0.6856 to 1.7985 & 0.6719 \\
\hline CEA & -0.0006 & 0.0015 & 0.1377 & 0.9994 & 0.9964 to 1.0024 & 0.7106 \\
\hline CRP & 0.0006 & 0.0029 & 0.0462 & 1.0006 & 0.9950 to 1.0062 & 0.8299 \\
\hline $\mathrm{LDH}$ & -0.0001 & 0.0007 & 0.0137 & 0.9999 & 0.9985 to 1.0014 & 0.9069 \\
\hline \multicolumn{7}{|l|}{ Multivariate } \\
\hline NLR & 0.0856 & 0.0333 & 6.6053 & 1.0894 & 1.0209 to 1.1625 & 0.0102 \\
\hline KPS (80-90 vs 100) & 0.5446 & 0.2412 & 5.0999 & 1.7239 & 1.0772 to 2.7590 & 0.0239 \\
\hline ESR & 0.0113 & 0.0084 & 1.8038 & 1.0114 & 0.9949 to 1.0282 & 0.1793 \\
\hline d-dimer & 0.0000 & 0.0001 & 0.4793 & 1.0000 & 0.9999 to 1.0002 & 0.4887 \\
\hline
\end{tabular}

100) also had a statistically significant association with survival. Variables associated with $\mathrm{p}$ value $<0.05$ at the univariate analysis were also evaluated in a multivariate Cox-regression model. Among all the selected factors, only KPS and NLR were independently associated with survival, with NLR being the most powerful independent prognosticator (exp(b) $1.09,95 \% \mathrm{CI}$ 1.02-1.16, p 0.01) (Table 2).

\subsection{Predictive value of NLR}

To assess whether NLR would influence the efficacy of oxaliplatin when added to gemcitabine, the effect of the type of chemotherapy on OS was analysed separately in patients with low and high baseline NLR. A significant interaction for OS between NLR and type of chemotherapy was found (test for interaction $\mathrm{p}$-value $\leqslant 0.0001$ ), with no difference between GEM and GEMOXA in the low NLR subgroup (mOS 11.8 vs 11.7 months, respectively, p 0.26, Fig. 3A). Conversely, the benefit of GEMOXA doublet was confined only to patients with NLR $>2.5$, with a mOS of 9.7 and 3.9 months for GEMOXA vs GEM, respectively, HR 2.08, 95\%CI 1.20-3.61, p 0.005 (Fig. 3B).
Moreover, the change of NLR during the first month of chemotherapy correlated with treatment activity, in particular a reduction of NLR was associated with a higher DCR (50\% vs $28 \%$, respectively) as determined at the first CT scan reassessment after three months of therapy, with an Odds Ratio of 0.39, p 0.02. The proportion of patients with NLR reduction after chemotherapy was $45 \%$ and $53 \%$ for GEM and GEMOXA, respectively (chi-squared test p 0.468).

In further confirmation of this, among patients with high baseline NLR, those whose NLR decreased below the cutoff value of 2.5 during chemotherapy had a significantly longer survival than patients who maintained NLR $>2.5$ (mOS for patients with low baseline NLR, NLR changing from $>2.5$ to $<2.5$ and persistent NLR $>2.5$ of 9.2, 7.7 and 5.0 months, respectively, p 0.003, Fig. 4).

\section{Discussion}

In the present study we were able to confirm NLR as a powerful prognostic biomarker in a homogenous set of metastatic PDA patients consisting of 


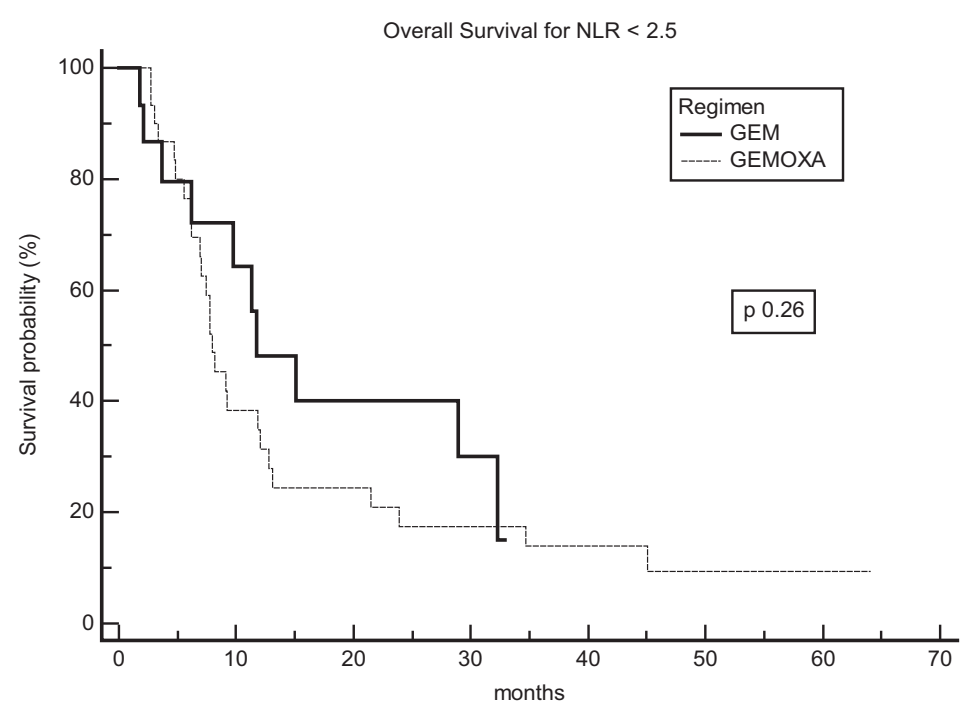

A

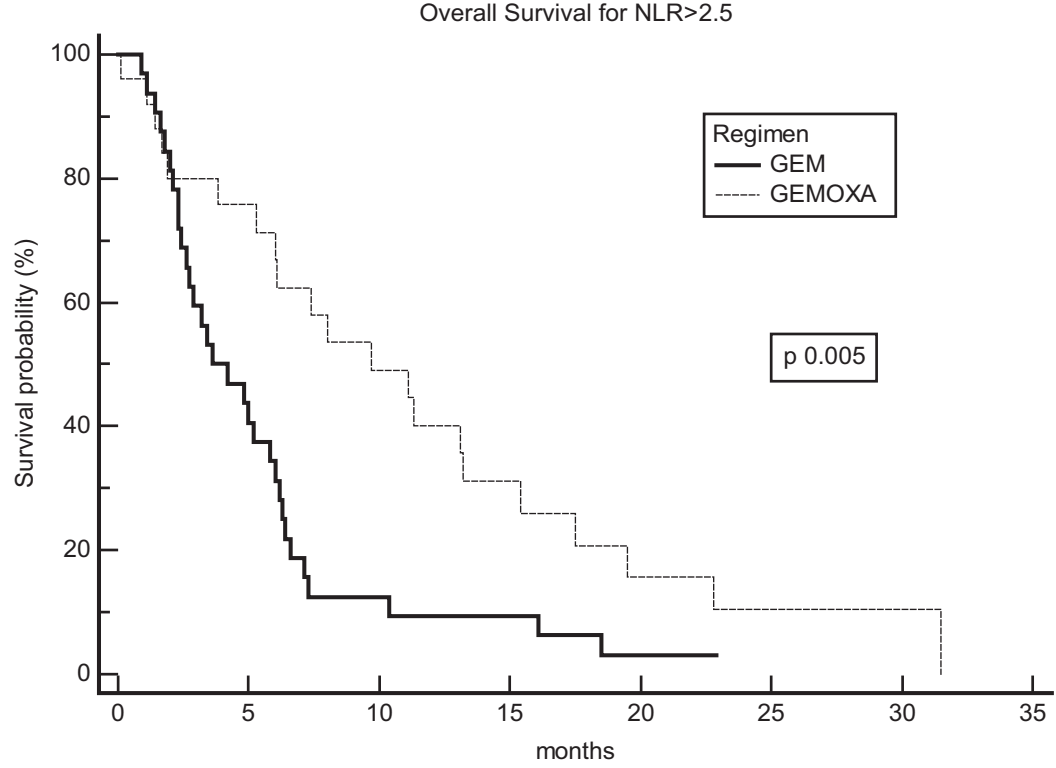

B

Fig. 3. Effect of chemotherapy on survival in low $(<2.5)(\mathrm{A})$ and high $(\geqslant 2.5)(\mathrm{B})$ baseline neutrophil/lymphocyte ratio (NLR) patients. GEM: gemcitabine monotherapy. GEMOXA; gemcitabine plus oxaliplatin.

two subgroups, the GEM monotherapy group and the GEMOXA group.

Authors acknowledge that this is a single retrospective study and the population enrolled is quite small. Nonetheless, to the best of our knowledge, this is the first report analyzing the predictive role of NLR with regards to oxaliplatin activity in PDA patients. Moreover, we highlighted the clinical relevance of NLR when assessed longitudinally after one month of treatment.
In the whole population, GEMOXA was superior to GEM in terms of OS, with borderline p value (Fig. 1). It cannot be excluded that larger sample size would make significant the GEMOXA superiority in an unselected PDA population. However, two phase III randomized trials (the E6201 and the GERCOR/GISCAD trial, $n=832$ and 326 , respectively), specifically testing this hypothesis, have failed to reach statistical significance $[5,8]$, thus confirming that oxaliplatin may be effective only in a select group of patients. In our uni- 


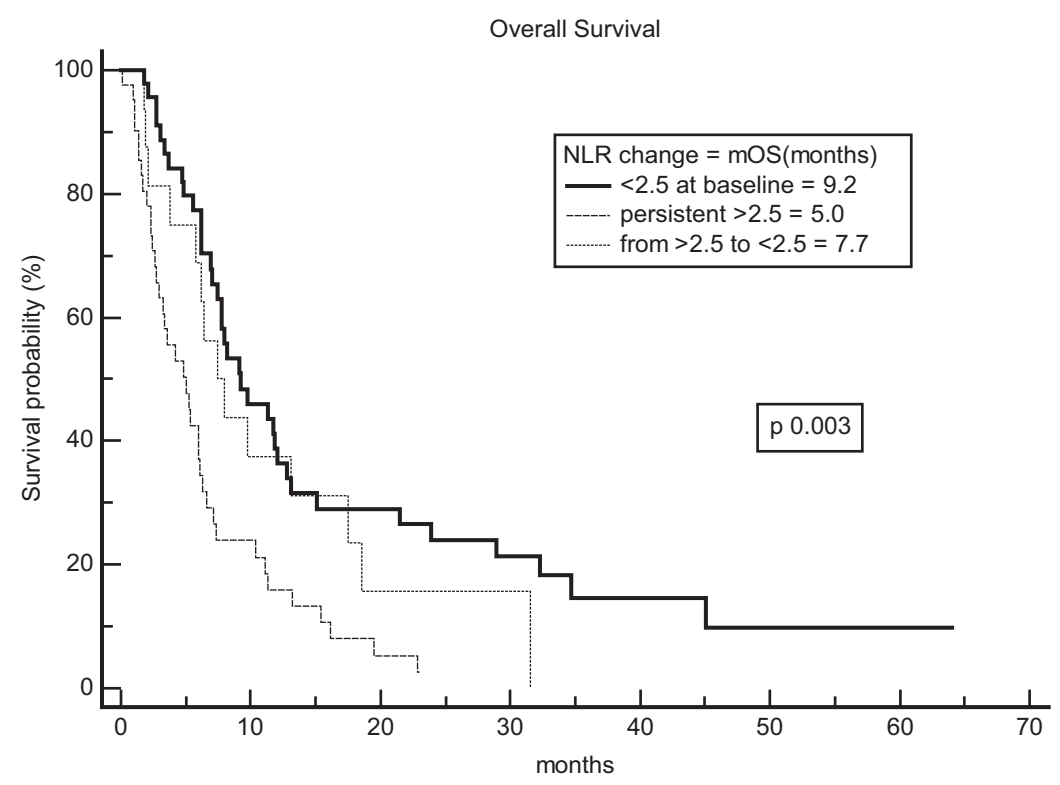

Fig. 4. Overall Survival (OS) and neutrophil/lymphocyte ratio (NLR) change during chemotherapy. mOS: median OS.

variate Cox-regression analysis, the type of chemotherapy was not significantly associated with survival (Table 2). Type of chemotherapy remained not significant even when included in a multivariate analysis together with NLR, KPS, ESR and d-dimer (data not shown). According to our analysis, NLR was the best predictive factor of oxaliplatin efficacy in that GEMOXA was superior to GEM only in the high baseline NLR group.

Furthermore, NLR reduction during chemotherapy correlated with disease control rate (DCR 50\% v 28\%, p 0.02), and patients with high baseline NLR in whom this variable decreased below the cutoff value of 2.5 during treatment had a survival comparable to that of patients with favourable features.

It can be hypothesized that patients with better prognosis $(\mathrm{NLR}<2.5)$ are more likely to receive oxaliplatin as second-line therapy after gemcitabine monotherapy failure, thus resulting in comparable survival to patients treated with GEMOXA upfront. Conversely, tumors with a more aggressive behaviour (NLR > 2.5) may have a rapid progression on first-line monotherapy (mOS 3.9 months) and second line administration may be precluded. In our cohort, in fact, among GEM monotherapy patients, $48 \%$ of those with NLR $<2.5$ received a second-line therapy, as compared to $8 \%$ of those with NLR $>2.5$ (data not shown). In these patients an intensive combination chemotherapy may be more appropriate in the first-line setting.

A number of studies have evaluated the prognostic value of NLR, first in patients with localized PDA candidate for radical surgery and subsequently also in large cohorts including metastatic patients treated with chemotherapy. Following a thorough PubMed search, we found two studies on localized PDA and five studies on metastatic PDA that we thought to be of particular interest.

In a study by Hamed et al., 228 patients with tumor of the periampullary region (pancreatic cancer, ampullary cancer or cholangiocarcima) undergoing pancreatoduodenectomy were retrospectively reviewed. Median survival of patients with NLR $<5$ was 24 months, whilst patients with NLR $>5$ had a mOS of 13 months. NLR maintained its prognostic value also among patients with disease relapse [29]. In another dataset by Garcea et al., NLR significantly predicted disease free survival (DFS) among radically resected patients with pancreatic cancer. Median DFS was 12 and 52 months for patients with preoperative NLR > or $<5$, respectively, $p<0.001$ [30].

Szkandera et al. reported, in two different papers, the analysis of a large cohort of PDA patients ( $n=$ $474)$, most of whom $(n=344)$ received palliative chemotherapy for a stage IV disease. NLR was confirmed to be an independent prognostic biomarker in multivariate analysis, together with tumor stage, grade, administration of chemotherapy and high CRP [31]. NLR cut-off was initially set at 5, according to the previous literature. Later, a ROC analysis was performed and a cut-off value similar to that obtained in our analysis was identified (i.e. NLR 2.3) [32]. 
Xue et al. have recently reported on 252 Japanese patients with advanced PDA receiving palliative chemotherapy, mainly GEM (70\%). No patients received GEMOXA. NLR was confirmed to be an independent prognostic factor. Median survival was longer than that observed in our cohort, maybe owing to the difference in ethnicity (mOS 13.4 and 8.6 months for NLR $>$ and $\leqslant 3$, respectively, $p<0.01$ ) [33].

Other two studies have investigated the prognostic value of NLR in PDA patients treated with gemcitabine -based chemotherapy. In a study by An et al., 95 patients were enrolled, $18 \%$ had NLR $>5$ and this was associated with a median survival of 2.4 months as compared to 7.7 months for NLR $<5, p<0.001$ [34]. Almost half of the patients received GEMOXA (44\%), but the possible predictive value of NLR for GEMOXA activity was not analysed. In another dataset by Teo et al., 85 patients (of whom only three patients received GEMOXA) were analysed and NLR $>3$ was associated to $\mathrm{mOS}$ of 3.4 , as compared to 9.4 for patients with NLR $<3, p=0.001$ [35]. Median survival in these two reports is comparable to that obtained in our study.

More recently, Luo et al. have confirmed the prognostic value of both baseline NLR and NLR change induced by chemotherapy in 403 patients with advanced pancreatic adenocarcinoma treated with different regimens of chemotherapy. NLR cutoff value was set at 3.1 [36]. NLR was an independent predictor in a multivariable model including serum CA19-9 levels and serum albumin levels. Albumin levels are often analyzed in conjunction with NLR and are included in an inflammatory index known as the Glasgow Prognostic Score (GPS) [37]. In our cohort the variables on which GPS is based (i.e. CRP and albumin) were not significantly associated with survival (Table 2).

We recognize a number of limitations in our study. First of all, as already mentioned, the research was retrospective and sample size was relatively small. Moreover, the analysis of the predictive value of NLR for oxaliplatin efficacy was achieved by dividing further the population in smaller treatment subgroups. Treatment assignment was not carried out upon randomization and this is a major drawback. Even though type of regimen was chosen by criteria other than PS, and the proportion of patients with KPS 80-90 was similar in the GEM and GEMOXA group, a selection bias cannot be ruled out. Randomized trials investigating prospectively the prognostic/predictive value of NLR would be desirable. Moreover the choice of GEMOXA as the preferred doublet in our Institution can be arguable and the patients' preference for GEM, mainly based on its less toxicity, can be considered 'too subjective' from a methodological point of view. Finally, we had no blood sample available to assess in depth the systemic inflammatory response in our cohort. In particular, some mediators are known to drive the systemic inflammation in cancer patients and may help clarify the biological mechanisms underlying our findings. As an example, circulating cytokines, such as IL-6 and TNF, should be included in future prospective trials analyzing NLR in this setting of patients [38,39].

In conclusion, in light of two recent phase III trials demonstrating the superiority of new combination regimens (i.e. FOLFIRINOX and nab-paclitaxel + gemcitabine) over gemcitabine monotherapy, but with higher toxicity, NLR may represent a readily available and inexpensive test to select patients for whom a particularly poor prognosis would justify the use of these more intensive, yet less tolerable, combination chemotherapy.

\section{Acknowledgement}

We thank Dr. Christine Tracey for revising the English and contributing to the clarity of the information provided in the present article.

\section{Conflict of interest}

Authors have no conflict of interest to declare.

\section{References}

[1] Hamada T, Nakai Y, Yasunaga H, Isayama H, Matsui $H$, Takahara N, Sasaki T, Takagi K, Watanabe T, Yagioka H, Kogure H, Arizumi T, Yamamoto N, Ito Y, Hirano K, Tsujino T, Tada M and Koike K, Prognostic nomogram for nonresectable pancreatic cancer treated with gemcitabine-based chemotherapy, Br J Cancer 110 (2014), 1943-1949.

[2] Seufferlein T, Bachet JB, Van Cutsem E and Rougier P, ESMO Guidelines Working Group. Pancreatic adenocarcinoma: ESMO-ESDO Clinical Practice Guidelines for diagnosis, treatment and follow-up, Ann Oncol 23(Suppl 7) (2012), vii33-40.

[3] Berlin JD et al., Phase III study of gemcitabine in combination with fluorouracil versus gemcitabine alone in patients with advanced pancreatic carcinoma: Eastern Cooperative Oncology Group Trial E2297, J Clin Oncol 20 (2002), 3270-3275.

[4] Rocha Lima CM et al., Irinotecan plus gemcitabine results in no survival advantage compared with gemcitabine monotherapy in patients with locally advanced or metastatic pancreatic cancer despite increased tumor response rate, J Clin Oncol 22 (2004), 3776-3783. 
[5] Louvet $\mathrm{C}$ et al., Gemcitabine in combination with oxaliplatin compared with gemcitabine alone in locally advanced or metastatic pancreatic cancer: Results of a GERCOR and GISCAD phase III trial, J Clin Oncol 23 (2005), 3509-3516.

[6] Heinemann V et al., Randomized phase III trial of gemcitabine plus cisplatin compared with gemcitabine alone in advanced pancreatic cancer, J Clin Oncol 24 (2006), 3946-3952.

[7] Herrmann R et al., Gemcitabine plus capecitabine compared with gemcitabine alone in advanced pancreatic cancer: a randomized, multicenter, phase III trial of the Swiss Group for Clinical Cancer Research and the Central European Cooperative Oncology Group, J Clin Oncol 25 (2007), 2212-2217.

[8] Poplin E et al., Phase III, randomized study of gemcitabine and oxaliplatin versus gemcitabine (fixed-dose rate infusion) compared with gemcitabine (30-minute infusion) in patients with pancreatic carcinoma E6201: A trial of the Eastern Cooperative Oncology Group, J Clin Oncol 27 (2009), 3778-3785.

[9] Kindler HL et al., Gemcitabine plus bevacizumab compared with gemcitabine plus placebo in patients with advanced pancreatic cancer: phase III trial of the Cancer and Leukemia Group B (CALGB 80303), J Clin Oncol 28 (2010), 3617 3622.

[10] Colucci G et al., Randomized phase III trial of gemcitabine plus cisplatin compared with single-agent gemcitabine as first-line treatment of patients with advanced pancreatic cancer: the GIP-1 study, J Clin Oncol 28 (2010), 1645-1651

[11] Cunningham D et al., Phase III randomized comparison of gemcitabine versus gemcitabine plus capecitabine in patients with advanced pancreatic cancer, J Clin Oncol 27 (2009), 5513-5518.

[12] Philip PA et al., Phase III study comparing gemcitabine plus cetuximab versus gemcitabine in patients with advanced pancreatic adenocarcinoma: Southwest Oncology Group-directed intergroup trial S0205, J Clin Oncol 28 (2010), 3605-3610.

[13] Sultana A, Smith CT, Cunningham D, Starling N, Neoptolemos JP and Ghaneh P, Meta-analyses of chemotherapy for locally advanced and metastatic pancreatic cancer, J Clin Oncol 25 (2007), 2607-2615.

[14] Conroy T, Desseigne F, Ychou M, Bouché O, Guimbaud R and Bécouarn Y, Groupe Tumeurs Digestives of Unicancer; PRODIGE Intergroup. FOLFIRINOX versus gemcitabine for metastatic pancreatic cancer, N Engl J Med 364 (2011), 18171825 .

[15] Goldstein D, El-Maraghi RH, Hammel P, Heinemann V, Kunzmann V and Sastre J, nab-Paclitaxel plus gemcitabine for metastatic pancreatic cancer: Long-term survival from a phase III trial, J Natl Cancer Inst 107 (2015), pii.

[16] Bellia A, Garcovich C, D'Adamo M, Lombardo M, Tesauro $\mathrm{M}$ and Donadel G, Serum 25-hydroxyvitamin D levels are inversely associated with systemic inflammation in severe obese subjects, Intern Emerg Med 8 (2013), 33-40.

[17] Brevetti G, Giugliano G, Brevetti L and Hiatt WR, Inflammation in peripheral artery disease, Circulation 122 (2010), 1862-1875.

[18] Redlich K and Smolen JS, Inflammatory bone loss: Pathogenesis and therapeutic intervention, Nat Rev Drug Discov 11 (2012), 234-250.

[19] Guadagni F, Ferroni P, Palmirotta R, Del Monte G, Formica V and Roselli M, Non-steroidal anti-inflammatory drugs in cancer prevention and therapy, Anticancer Res 27 (2007), 31473162 .

[20] Guadagni F, Ferroni P, Palmirotta R, Portarena I, Formica $\mathrm{V}$ and Roselli M, Review. TNF/VEGF cross-talk in chronic inflammation-related cancer initiation and progression: An early target in anticancer therapeutic strategy, In Vivo $\mathbf{2 1}$ (2007), 147-161.

[21] Formica V, Cereda V, di Bari MG, Grenga I, Tesauro M and Raffaele P, Peripheral CD45RO, PD-1, and TLR4 expression in metastatic colorectal cancer patients treated with bevacizumab, fluorouracil, and irinotecan (FOLFIRI-B), Med Oncol 30 (2013), 743.

[22] Formica V, Cereda V, Nardecchia A, Tesauro M and Roselli $\mathrm{M}$, Immune reaction and colorectal cancer: friends or foes? World J Gastroenterol 20 (2014), 12407-12419.

[23] Formica V, Luccchetti J, Cunningham D, Smyth EC, Ferroni $\mathrm{P}$ and Nardecchia A, Systemic inflammation, as measured by the neutrophil/lymphocyte ratio, may have differential prognostic impact before and during treatment with fluorouracil, irinotecan and bevacizumab in metastatic colorectal cancer patients, Med Oncol 31 (2014), 166

[24] Sanjay P, de Figueiredo RS, Leaver H, Ogston S, Kulli C and Polignano FM, Preoperative serum C-reactive protein levels and post-operative lymph node ratio are important predictors of survival after pancreaticoduodenectomy for pancreatic ductal adenocarcinoma, JOP 13 (2012), 199-204.

[25] Hess V et al., CA 19-9 tumour-marker response to chemotherapy in patients with advanced pancreatic cancer enrolled in a randomised controlled trial, Lancet Oncol 9 (2008), 132-138.

[26] Stocken DD et al., Modelling prognostic factors in advanced pancreatic cancer, Br J Cancer 99 (2008), 883-893.

[27] Liu P, Zhu Y, Liu L et al., Elevated pretreatment plasma Ddimer levels and platelet counts predict poor prognosis in pancreatic adenocarcinoma, Onco Targets Ther 8 (2015), 13351340 .

[28] Shiny A, Bibin YS, Shanthirani CS, Regin BS, Anjana RM and Balasubramanyam M, Association of NeutrophilLymphocyte Ratio with Glucose Intolerance: An Indicator of Systemic Inflammation in Patients with Type 2 Diabetes, $D i$ abetes Technol Ther 16 (2014), 524-30.

[29] Hamed MO, Roberts KJ, Smith AM and Morris Stiff G, Elevated pre-operative neutrophil to lymphocyte ratio predicts disease free survival following pancreatic resection for periampullary carcinomas, Pancreatology 13 (2013), 534-538.

[30] Garcea G, Ladwa N, Neal CP, Metcalfe MS, Dennison AR and Berry DP, Preoperative neutrophil-to-lymphocyte ratio (NLR) is associated with reduced disease-free survival following curative resection of pancreatic adenocarcinoma, World J Surg 35 (2011), 868-872.

[31] Szkandera J, Stotz M, Eisner F, Absenger G, Stojakovic T and Samonigg H, External validation of the derived neutrophil to lymphocyte ratio as a prognostic marker on a large cohort of pancreatic cancer patients, PLoS One 8 (2013), e78225.

[32] Szkandera J, Stotz M, Absenger G, Stojakovic T, Samonigg $\mathrm{H}$ and Kornprat $\mathrm{P}$, Validation of C-reactive protein levels as a prognostic indicator for survival in a large cohort of pancreatic cancer patients, Br J Cancer 110 (2014), 183-188.

[33] Xue P, Kanai M, Mori Y, Nishimura T, Uza N and Kodama Y, Neutrophil-to-lymphocyte ratio for predicting palliative chemotherapy outcomes in advanced pancreatic cancer patients, Cancer Med 3 (2014), 406-415.

[34] An X, Ding PR, Li YH, Wang FH, Shi YX and Wang ZQ, Elevated neutrophil to lymphocyte ratio predicts survival in advanced pancreatic cancer, Biomarkers 15 (2010), 516-522.

[35] Teo M, Mohd Sharial MS, McDonnell F, Conlon KC and Ridgway PF, Prognostic role of neutrophil-to-lymphocyte ratio in advanced pancreatic ductal adenocarcinoma: impact of baseline fluctuation and changes during chemotherapy, Tumori 99 (2013), 516-522. 
[36] Luo G, Guo M, Liu Z, Xiao Z, Jin K, Long J, Liu L, Liu C, Xu $\mathrm{J}, \mathrm{Ni} \mathrm{Q}$ and $\mathrm{Yu} \mathrm{X}, \mathrm{Blood}$ neutrophil-lymphocyte ratio predicts survival in patients with advanced pancreatic cancer treated with chemotherapy, Ann Surg Oncol 22 (2015), 670-676

[37] Stotz M, Gerger A, Eisner F, Szkandera J, Loibner H and Ress $\mathrm{AL}$, Increased neutrophil-lymphocyte ratio is a poor prognostic factor in patients with primary operable and inoperable pancreatic cancer, Br J Cancer 109 (2013), 416-421.
[38] Clarke S, Burge M, Cordwell C, Gibbs P, Reece W and Tebbutt N, An Australian translational study to evaluate the prognostic role of inflammatory markers in patients with metastatic ColorEctal caNcer Treated with bevacizumab (Avastin ${ }^{\mathrm{TM}}$ ) [ASCENT], BMC Cancer 13 (2013), 120.

[39] Kantola T, Klintrup K, Väyrynen JP, Vornanen J, Bloigu $\mathrm{R}$ and Karhu T, Stage-dependent alterations of the serum cytokine pattern in colorectal carcinoma, $\mathrm{Br} J$ Cancer 107 (2012), 1729-1736. 
Copyright of Cancer Biomarkers is the property of IOS Press and its content may not be copied or emailed to multiple sites or posted to a listserv without the copyright holder's express written permission. However, users may print, download, or email articles for individual use. 